\title{
Diagnose, Prognose, Prädiktion
}

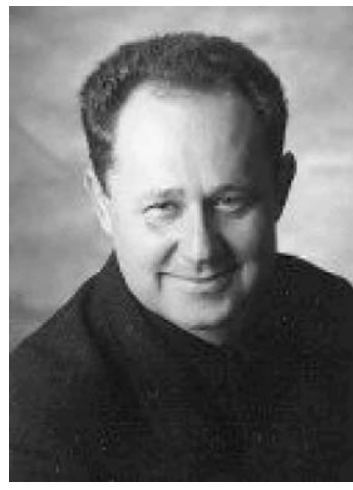

Prof. Dr. med. Manfred Wildner
Bibliografie

DOI http://dx.doi.org/

10.1055/s-0034-1372569

Gesundheitswesen 2014;

76: $185-186$

(c) Georg Thieme Verlag KG

Stuttgart · New York

ISSN 0941-3790

Korrespondenzadresse

Prof. Dr. med. Manfred Wildner

Bayerisches Landesamt für Gesundheit und Lebensmittelsicherheit

Veterinärstraße 2

85764 Oberschleißheim

manfred.wildner@lgl.bayern.de
Der wohl größte Beitrag der hippokratischen Ärzteschule für unsere heutige gesundheitliche Versorgung war die Grundlegung einer empirisch-rationalen Medizin. Der Gedanke, durch aufmerksame Beobachtung einer ausreichend großen Zahl von Krankheitsverläufen eine Voraussage auch für andere Patienten machen zu können, war durchaus revolutionär. Er mündete in den hippokratischen Dreiklang von Diagnose, Prognose und Therapie. In dieser Reihenfolge? Die hippokratische Prognose war als Vorhersage des wahrscheinlichen Krankheitsverlaufs zentraler Bestandteil der ärztlichen Kunst. Sie stand als Grundlage der Therapie vor allem vor, nicht nach der Therapieentscheidung [1]. In Abwandlung der bekannten Formulierung des Schweizer Internisten Otto Naegeli „Vor die Therapie setzten die Diagnose die unsterblichen Götter“ [2] ließe sich also auch sagen „Vor die Therapie setzten die Prognose die unsterblichen Götter". Beides in Analogie zum Vers des altgriechischen Philosophen Hesiod: Vor das Gedeihen jedoch haben die ewigen Götter den Schweiß gesetzt [3].

Neben die Prognose ist in der Moderne leise ein weiterer Begriff getreten: Die Prädiktion. Der feine Unterschied zwischen Prädiktion und Prognose verdient Aufmerksamkeit: Während Prognose eine Vorausschau über den wahrscheinlichen Verlauf einer bestehenden Krankheit geben will, auch unter Berücksichtigung von therapeutischen Optionen, setzt die Prädiktion schon beim gesunden Menschen an. Sie ist im Rahmen von Lebensversicherungen und Dienstfähigkeitsbeurteilungen bereits bekanntes Gedankengut. Dabei steht, neben der Prognose bestehender Krankheiten, die Beurteilung von individuellen Risikofaktoren wie Body Mass Index oder Blutdruck und - bei Lebensversicherungen erst ab Versicherungssummen in größerer Höhe - auch von genetischen Merkmalen im Mittelpunkt. Sie ist Grundlage für die Prämienfestsetzung bei Versicherungsabschluss oder die Feststellung der beruflichen gesundheitlichen Eignung.

Welche weiteren handlungsrelevanten Konsequenzen ergeben sich aus den Vorhersagen zu den Auswirkungen der so festgestellten persönlichen Risikofaktoren, für sich genommen und in ihren Wechselwirkungen? Die Vorhersage der individuellen Reaktionsweise auf Medikamente ist eine der großen Hoffnungen einer personalisierten bzw. individualisierten Medizin: Eine nebenwirkungsärmere, weil individualisierte Pharmakotherapie, womöglich weiterentwickelt zum gewebespezifischen Drug-Delivery-System. Eine präventive Lebensstilintervention in Abhängigkeit von den individuell gegebenen Gesundheits- risiken wäre ein weiterer, ebenfalls ehrgeiziger Ansatzpunkt.

Eine solche „Personalisierte Medizin“, wie sie gegenwärtig verstanden wird, zielt auf Differenzierung, auf den Einzelfall anvisierende Methoden. Weitgehend synonyme Bezeichnungen sind individualisierte, stratifizierte, prädiktive oder Präzisions-Medizin. Sie hat ihre Wurzeln in der Molekularbiologie und den damit verbundenen OMICWissenschaften: Genomics, RNAomics, Epigenomics, Proteomics. Leroy Hood, US-amerikanischer Molekularbiologe und Mitbegründer des Instituts für Systembiologie, prägte 2004 das Konzept der P4-Medizin: Einer prädiktiven, präventiven, personalisierten und partizipativen Medizin der Zukunft, Folge eines molekularbiologisch getriggerten Paradigmenwechsels der modernen Medizin [4,5].

Die Prinzipien einer solchen prädiktiven Medizin sind dabei bereits jetzt in der Versorgungspraxis gegenwärtig: Beispiele sind genetische Untersuchungen hinsichtlich eines klinisch asymptomatischen Carrier-Status bei auffälliger familiärer Anamnese oder auch vertraute nicht-genetische Verfahren wie die einfache Blutdruckmessung als mögliche Risikofaktorbestimmung für Herz-Kreislauf-Erkrankungen oder die Erfassung Diabetesspezifischer Risikofaktoren. Diese prädiktiven Verfahren sind in ihrer (sekundär-)präventiven Ausrichtung auf die proaktive Mitwirkung der Patienten vor Ausbruch der Krankheit angewiesenen: Kernelement der „partizipatorischen“ Medizin.

Personalisierte Medizin - nur alter Wein in neuen Schläuchen? Es bestehen durchaus Besonderheiten der neuen, „personalisierten“ P4-Medizin. Bei vielen Analyseergebnissen der OMIC-Analysen handelt sich um „Zitate“ aus unseren persönlichen genetischen Bauplänen oder um epigenetische „Fußnoten“ dazu. Damit ist also nicht mehr die Rede von äußeren Wirkursachen, wie das z.B. bei der genetischen Analyse von mikrobiologischen Krankheitserregern der Fall wäre. Vielmehr ist die Rede von inneren Dispositionen und Suszeptibilitäten, sogenannten Materialursachen [6]. Diese sind zunächst keine Krankheitserscheinungen, sondern lediglich Indikatoren persönlicher biologischer, im Sinne der Gen-Umwelt-Interaktion teilweise auch biografischer Gegebenheiten. Die diagnostischen Optionen scheinen dabei schneller verfügbar als die therapeutischen. Firmen wie Genetics Technology, Phenogen Sciences oder 23andMe entfalten ihre diesbezüglichen Aktivitäten auf dem Gesundheitsmarkt. In den globalisierten Weiten des Internets sind personalisierte Genomuntersuchungen bereits Realität. Teilweise wird ein direkt käufliches Testangebot ohne Zugangsbeschränkung gemacht, in 
Form von spezifischen DNA Arrays oder auch als vollständige Analyse des persönlichen Erbgutes („full genom sequencing“). So bietet eine Firma für wenig Geld Gesundheitsberichte über hunderte von möglichen Gesundheitsgefährdungen und Veranlagungen an, zusätzlich Informationen zu Verwandtschaftsverhältnissen sowie zur wahrscheinlichen Reaktibilität auf Medikamente [7].

Diese „persönlichen“ Befunde lassen erst nach prädiktiver Interpretation "personalisierte“ diagnostische und therapeutische Aussagen zu, z.B. zu einer Arzneimitteltherapie oder empfohlenen Lebensweise. Man sollte sich dabei vor einer medikalisierenden bzw. pathologisierenden Sichtweise hüten, welche genetische Materialursachen einseitig als Defekt interpretiert. Und welche womöglich eine Genotyp-Prävention an Stelle einer verhaltens- oder verhältnisorientierten Phänotyp-Prävention setzt und damit einer Verlagerung von Gesundheit und Gesunden zu Krankheit und Kranken Vorschub leistet [8].

Bei den schönen, teilweise schön gefärbten Zukunftshoffnungen der P4-Medizin gilt also: Nicht jedes „P“ ist ohne Abstriche positiv zu sehen. Was ist bspw. mit den Fällen, in welchen keine perfekte prädiktive Aussage gemacht werden kann? Die Ursachen können in der polygenen Natur einer Erkrankung liegen oder auch in einer Variabilität der Ausprägung von genetischen Sequenzen als Proteine. Was ist, wenn eine Prädiktion zwar möglich ist, eine Prävention aber ausscheidet? Was ist mit Fällen, welche ähnlich wie das oft klinisch stumme Prostatakarzinom beim Mann zu bewerten sind, welches häufig erst posthum diagnostiziert wird? Wer leistet die notwendigen sachkundigen und objektiven individuellen Beratungen, wer übernimmt die gesellschaftliche Informations- und Diskursverantwortung? Ergeben sich möglicherweise politische Implikationen durch die Ausgrenzung von Genträgern oder auch Bevölkerungsgruppen von bestimmten professionellen Tätigkeiten aufgrund ihres Risikoprofils - eine genetische Klassengesellschaft als Spielform einer „Schönen neuen Welt“ (Aldous Huxley)?

Individuelle Gesundheitsdaten mit prädiktivem Charakter haben ohnehin Konjunktur: Die "Quantified Self"-Bewegung nimmt, als weitere Spielform, sehr konkret eine umfassende Digitalisierung der verfügbaren Daten zu Umwelt und eigenem Körper in Angriff. Durch ein solches intensives Self-Tracking sollen individuelle Lösungen für persönliche Fragestellungen in Sport, Gesundheit und anderen Lebensbereichen gefunden werden (www.quantifiedself.com). Sehen wir ein Empowerment als Befähigung zur Eigenverantwortung oder doch nur eine neue Fixierung auf Expertenwissen? Reicht die Summe der individuellen Gesundheitsinteressen für eine gesundheitsförderliche Gestaltung der gesundheitsrelevanten Lebenswelten? Noch ist die Sicht unklar.

Vielfältige rechtliche, gesellschaftliche und ethische Erwägungen sind mit der P4-Medizin verbunden. Geführt werden solche Diskurse bereits: Seitens der Deutschen Gesellschaft für Medizinrecht (Einbecker Empfehlungen), von der Deutschen Gesellschaft für Humangenetik (Stellungnahme zu genetischen $\mathrm{Zu}-$ satzbefunden in Diagnostik und Forschung), vom Nationalen Ethikrat (Nutzung von prädiktiven Gesundheitsinformationen bei Einstellungsuntersuchungen), in medizinethischen Diskussionsforen, als gemeinsame Stellungnahme wissenschaftlicher Fachgesellschaften [9]. Auf europäischer Ebene wurde das Public Health Genomic European Network (www.phgen.eu) mit EUMitteln gefördert. Von dem PHGEN-Netzwerk wurden u. a. differenzierte Best Practice Guidelines für die Qualitätssicherung, die Bereitstellung und die Nutzung von Genombasierter Information und zugehörigen Technologien erstellt.
Die Publikation von Forschungsergebnissen zu Innovationen und ihre begleitende, auch ethische Reflexion ist wesentliche Aufgabe unserer Zeitschrift. Themen im vorliegenden Heft sind die Beteiligung von Patienten an medizinischen Entscheidungen, die Förderung von Obst- und Gemüseverzehr bei Schulkindern, die Mundgesundheit von berufstätigen Frauen im Vergleich, die Patientenzufriedenheit bei onkologischen Erkrankungen, die hausärztliche Perspektive zur Darmkrebsfrüherkennung, der elektronische Mutter- bzw. Eltern-Kind-Pass, Kriterien für die Priorisierung medizinischer Leistungen, Auswirkungen des Referenzpreissystems bei Impfstoffen und, aus aktuellem Anlass, ein Diskussionsbeitrag zu einem sich abzeichnenden Paradigmenwechsel in der Bewertung der gesundheitlichen Eignung von neu einzustellenden Beamtinnen und Beamten. Besonders wird auch auf die Stellungnahme des Fachausschuss Psychiatrie des BVÖGD zur Novellierung der Gesetze zu Hilfen psychisch Erkrankter (PsychKG) in den Bundesländern in diesem Heft hingewiesen. Zudem enthält das Heft einen bekannten und doch immer wieder neuen Schwerpunkt: Die Abstracts des 64. ÖGDKongresses 15.-17. Mai 2014, Magdeburg, dem wieder viel Erfolg gewünscht sei!

Liegen die alte hippokratische Medizin und die hoffnungsfrohen neuen Handlungsfelder der P4 Medizin weit auseinander? Löst eine P4-Medizin die in sie gesetzten Hoffnungen ein, so wird sich ihr prädiktives und präventives Potenzial nicht in einer weiter entwickelten Arzneimitteltherapie erschöpfen. Die Kenntnis von persönlichen, prädiktiv als Risikofaktoren interpretierten Merkmalen soll auch neue Impulse für partizipative, proaktive, präventive Handlungsoptionen geben. Sowohl Verhaltensprävention als auch Verhältnisprävention vollziehen sich in intensiver Wechselwirkung mit dem natürlichen, sozio-ökonomischen und politisch-kulturellen Umfeld. Sie sind schon jetzt wichtiger Forschungsgegenstand „herkömmlicher“ Prävention und Gesundheitsförderung. Verbindungen einer solchen gesundheitsförderlichen Lebensführung zu den Texten des Corpus Hippocraticum lassen sich ebenfalls mühelos knüpfen. Ob vor die Prävention die unsterblichen Götter die Prädiktion gesetzt haben, bleibt offen. Anstrengung und Schweiß wird jedenfalls auch vor das Gelingen guter Prädiktion gesetzt sein.

\section{Literatur}

1 Ratschko KW. Die Krankheiten zu Zeiten des Hippokrates. SchleswigHolsteinisches Ärzteblatt 2011; 1: 56-59 URL http://www.aerzte blatt-sh.de/system/files/print-archiv/einzel/shae_20110156_medi zingeschichte_krankheiten_zu_zeiten_des_hippokrates_0.pdf aufgerufen am 28.02.2014

2 Naegeli 0 . Differentialdiagnose in der inneren Medizin. Leipzig: Thieme; 1937

3 Keck FS. Wer hat vor die Therapie die Diagnose gesetzt? Hessisches Ärzteblatt 2007; 6: 388 URL http://www.laekh.de/upload/Hess._Ae rzteblatt/2007/2007_06/2007_06_19.pdf aufgerufen am 28.02.2014

4 Hood L, Heath JR, Phelps ME et al. Systems Biology and New Technologies Enable Predictive and Preventative Medicine. Science 2004; 306: 640-643

5 Leroy Hood, MD, PhD. Co-founder and Chairman P4 Medicine institute http://p4mi.org/leroy-hood-md-phd aufgerufen am 28.02.2014

6 Wildner M. Aristotle and the human genome project. Lancet 2000; 356: 1360

7 Annas GJ, Sherman E. 23andMe and the FDA. N Engl J Med 2014 [Epub ahead of print]

8 Trojan A, Kuhn J. Prädiktive Medizin und individualisierte Medizin. In: BzGA, Hrsg. Leitbegriffe der Gesundheitsförderung. BzGA; Köln: 2011; 422-426

9 Deutsche Akademie der Naturforscher Leopoldina/Nationale Akademie der Wissenschaften et al. Stellungnahme prädiktive genetische Diagnostik als Instrument der Krankheitsprävention. Berlin: Deutsche Akademie der Naturforscher Leopoldina e.V.; 2010 